\title{
Stratégie d'instrumentation pour la gestion optimisée des ouvrages portuaires
}

\author{
Franck Schoefs $^{(\mathrm{a}),}$ Bruno Gerard ${ }^{(\mathrm{b}) \text {, }}$ \\ Pascal Casari, Luc Verdure ${ }^{(\mathrm{c})}$
}

(a)Maître de Conférences, Institut de Recherche en Génie Civil et Mécanique (Gém), UMR 6183, Faculté des Sciences de Nantes, BP 92208 - 44322 Nantes cedex 3, 02511255 22-24, schoefs@physique.univ-nantes.fr

(b)PDG, Société Oxand, 36 bis avenue Franklin Roosevelt, 77210 Avon/Fontainebleau, 33 (0)1 60395251 bruno.gerard@oxand.com

(c)Doctorant, GéM, luc.verdure@physique.univ-nantes.fr

\section{Résumé:}

Le suivi et la requalification des structures de génie civil suppose d'acquérir des informations sur leur comportement sous forme d'inspections, de mesures ou d'instrumentation. Cet article présente les enjeux d'une stratégie d'instrumentation efficace sur une partie d'un parc de structures portuaires. On montre en particulier les différents niveaux de redondances nécessaires et les technologies disponibles.

\section{Abstract :}

The survey and reassessment of existing structures is still a great challenge for the decision makers. Decision aid tools are available and are based mostly on the understanding of the in-service behaviour of structures. This paper focuses of the optimal monitoring strategy for wharf where uncertainties on the in-service behaviour are significant. Technologies selection and information redundancy are presented as major criteria for facilities management.

Mots-clés:stratégie d'instrumentation, ouvrages portuaires, inspection, maintenance, réparation.

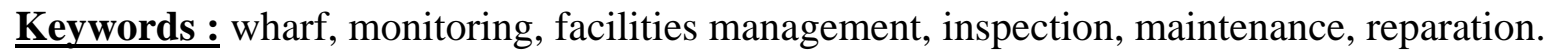

\section{Introduction}

Le suivi des structures de génie civil existantes nécessite de disposer d'un éventail de techniques et d'outils méthodologiques permettant une aide à la décision basée sur des outils rationnels tout en respectant l'expertise nécessaire. Parmi eux, on peut citer la collecte de données dans un objectif de retour d'expérience et la modélisation des phénomènes évolutifs (fatigue, corrosion).

Les structures portuaires rentrent dans ce cadre et sont exemplaires à trois titres liés à l'histoire et l'économie. La France a toujours su tirer profit de sa façade maritime exceptionnelle ; les ouvrages ont été construits de tout temps et on rencontre encore sur nos ports l'exploitation d'ouvrages dépassant les 100 ans. La seconde guerre mondiale a montré l'importance de la maîtrise et de la défense des côtes. Ceci a eu pour conséquence la réalisation de travaux de reconstruction ou de réhabilitation (cas du quai Wilson à Nantes). Enfin plus récemment, le développement du transport maritime a nécessité la construction de nouveaux ouvrages. 
Cette histoire est la cause de l'extrême diversité des ages et des techniques rencontrés sur les ouvrages portuaires rendant délicate une approche systématique de maintenance. De plus, les codes de calcul utilisés en conception ont parfois conduit à un sur-dimensionnement des structures, parfois à un mauvais dimensionnement. La complexité de ces ouvrages rend difficile la mise en rapport des règles utilisées à l'époque avec les exigences de sécurité actuelles.

L’article présente les éléments clés d'une stratégie d’inspection et d'instrumentation d’ouvrages portuaires basée sur une expérience menée avec le port autonome de Nantes Saint Nazaire au travers de 4 quais. Les enjeux de l'Instrumentation, Mesure, Inspection, Auscultation (I.M.I.A) sont présentés au niveau composant et structure. Ensuite les éléments clés d'une instrumentation orientée maintenance sont traités sans occulter les impératifs de chantier et technologiques notamment.

\section{Enjeux de l'IMIA pour le suivi des ouvrages}

\subsection{IMIA orientée composant}

Le suivi de composants structurels ou d'ouvrages entiers suppose la connaissance d'informations au long de l'exploitation de la structure mais aussi au moment de sa construction. A cette fin de nombreux outils sont disponibles :

-l'instrumentation de composants structurels,

-la mesure de grandeurs physiques par contrôles destructifs ou non (cas de la résistance du béton ou de carottages d'échantillons de sols) comme les déplacements mesurés directement (topographie) ou indirectement (laser),

-l'inspection d'éléments structurels en vue d'un diagnostic,

-l'auscultation d'éléments structurels en vue d'une analyse inverse notamment (cas de câbles de haubans de ponts).

Comme indiqué en figure 1, leur utilisation est fortement liés aux phases de construction et de service de l'ouvrage.

\begin{tabular}{|c|c|}
\hline Construction & Exploitation \\
\hline Instrumentation progressive & Instrumentation totale / complémentaire \\
\hline & Mesures \\
\hline & Auscultations / Inspections \\
\hline
\end{tabular}

Figure 1: Stratégie IMIA au cours de la vie d'un ouvrage

Afin de planifier une politique de Suivi-Maintenance-Réparation, l’information disponible doit être comparée à un niveau de référence donné voire à une performance souhaitée. Dans le cas d'une IMIA-composants, la note de calcul fournit une aide précieuse sur le niveau d'attente du concepteur. Toutefois, l'application des règles de l'Art et de Plans d'Application Qualité doit être scrupuleuse. Elle couvre notamment le traitement des non conformités et leur enregistrement. Pour certains ouvrages en liaison avec le sol, on doit analyser le caractère évolutif des mesures obtenues.

La collecte des données est donc plus importante et un recueil des « évènements et incidents de chantier » ainsi que des méthodes est nécessaire : phasage de remblaiement et de pose des tirants, avancement de la construction, zones de stockages, de passages d'engins, 
difficultés d'implantation, climatologie extrême ... Les acteurs du chantier doivent être sensibilisé à cet enregistrement de la qualité.

\subsection{IMIA orientée système}

Cet article se concentre sur la mise en place d'un suivi par instrumentation de structures portuaires dans l'objectif d'analyser leur comportement global. On parle alors d'IMIA système. Le recours à la note de calcul permet ici d'analyser l'ouvrage dans sa logique de conception. Toutefois, elle est moins utile dans une analyse système. En effet, les codes de calcul et les hypothèses successives visent à effectuer des conceptions conservatives. Les simplifications permettent notamment de contourner les phénomènes de couplage. Le fonctionnement réel d'une structure est donc d'autant plus éloigné que celui envisagé par la note de calcul que cette structure est complexe et sujette à des couplages avec le sol et l'eau notamment. C'est particulièrement le cas des quais sur pieux, en contact avec le sol au niveau des pieux, du mur d'arrière quai et des plaques des tirants d'ancrage et soumis à des chargements horizontaux importants et cycliques (amarrages, accostages, niveau de nappe associée à la marée, ...) qui mobilisent le sol. A titre d'exemple, les efforts de butée sur les murs d’arrière quai sont généralement calculés sans les surcharges dues aux enrochements.

\section{Instrumentation orientée maintenance}

On a vu ci-dessus la distinction nécessaire entre l'IMIA composant et l'IMIA système. Dans le cas d'une instrumentation orientée maintenance, on recherche le comportement en service de l'ouvrage en réalisant des mesures en des points représentatifs sans dégrader le comportement ou l’intégrité structuraux.

\subsection{Recherche de points représentatifs}

L'instrumentation fait appel à des techniques de mesure dans le contexte de fonctionnement d'un ouvrage. La notion d'incertitude sur les mesures doit être prise en compte notamment pour éviter que les mesures effectuées soient noyées dans cette incertitude. La note de calcul et le comportement prévu de l'ouvrage donnent des indications précieuses sur le niveau d'information qui peut être attendu. Une analyse de risque, même qualitative, permet ensuite de cerner les éléments à fort impact sur le comportement et ainsi de centrer l'analyse sur eux. La recherche de points sensibles et représentatifs du comportement peut alors être menée et le choix du capteur optimisé dans un triple objectif de coût/performance/fiabilité. Il faut cependant distinguer la notion de points ou composants représentatifs et de points sensibles. En effet, en instrumentation deux exigences doivent être respectées :

-la sensibilité de la mesure, permise par le capteur, aux différentes phases de comportement de l'ouvrage. Les composants sensibles instrumentés sont alors autant de capteurs du comportement de l'ouvrage.

-l'extrapolation des mesures sur des composants sensibles aux composants représentatifs intervenant dans des situations à risque. Des modèles de transport de l'information suffisamment fins doivent exister.

Cette situation se rencontre pour la mesure des déformations, extrêmement délicate pour des poutres à forte inertie présentant des chargements aléatoires. Dans ce cas les mesures doivent être reportées sur d'autres éléments. Pour les quais sur pieux, c'est le cas des poutres d'arrière quai ou d'accostage, très rigides et accolées à une plate-forme elle-même à forte inertie ; on préfère alors instrumenter les tirants d'ancrage ; voir ci-après les paragraphes 3.2 et 3.3 . 


\subsection{Prise en compte des exigences chantier}

L'instrumentation suppose la prise en compte du contexte du chantier ; les passages de chemins de câbles notamment sont autant de contraintes pour l'entreprise qui doit parfois les intégrer dans ses coûts, en terme de perturbation du phasage du chantier. Leur longueur et leur position doivent être étudiées afin d'éviter les arrachements et être évidemment compatible avec une acquisition en phase de chantier et en phase de service. De plus la présence de centrales d'acquisition provisoires sont autant de points à éviter par les engins. L'existence de mini-centrales portatives peu intrusives permet d'instrumenter un organe de la structure indépendamment de son environnement et de suivre les sollicitations qui lui sont appliquées durant toute la phase de chantier. Cette technique a été utilisée dans le cas du suivi du quai sur pieux (C) en phase de chantier (voir figure 2), pour le suivi de l'évolution des efforts dans des tirants d'ancrages au moyen de jauges de déformation. Seules les données des tirants 9 à 12 ne sont pas disponibles parce qu'ils n'étaient pas encore installés au 8 août.

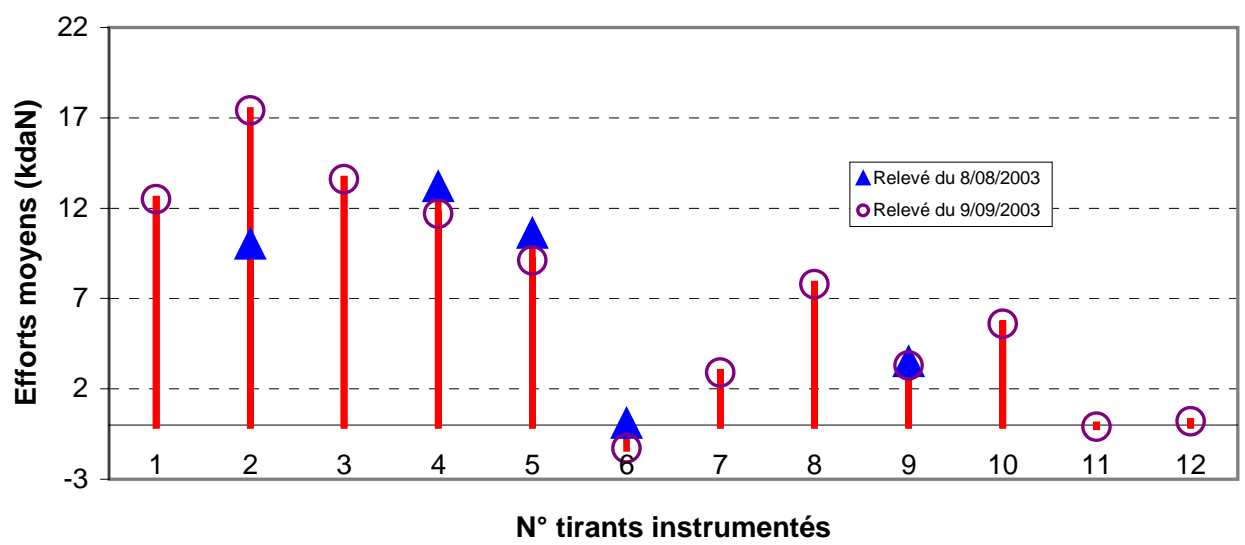

Figure 2: Suivi d'efforts dans des tirants en phase de chantier (quai C).

Deux relevés à deux dates ont été effectués à condition de marée similaire. Certains tirants sont encore très peu sollicités tandis que d'autres le sont déjà fortement: la cause n'est pas encore identifiée mais semble provenir d'un tassement du remblai généré par le passage répété d'engins pour l'approvisionnement en béton et en matériaux de remblai au niveau des tirants 1 à 7. Ce fait était dû à la présence d'une passerelle (information enregistrée par le conducteur de travaux) au droit des tirants 1 à 7. Pour un autre quai (T), la mise en place de cellule de charge (voir paragraphe3.3) sur chantier, avec mesure simultanée, a permis de mesurer une valeur à $100 \mathrm{kN}$ de l'effort de précontrainte créé par une pelleteuse. Une opération similaire réalisée sur un autre quai (D) afin d'éviter la flèche de tirants non remblayés a permis d'appréhender la dispersion sur ce genre de valeur et un coefficient de dispersion de $19 \%$ a été calculé (sur 30 tirants) sur les efforts introduits par l'engin de chantier.

\subsection{Recherche de capteurs éprouvés et transfert technologique}

On présente ci-dessous un comparatif de technologies de capteur dans le contexte particulier d'un quai sur pieux (T). L'objectif est la caractérisation du niveau d'effort repris par des tirants d'ancrage et leur comportement en temps que composant. On se limite dans cette section au comportement composant, le comportement système étant abordé au 
paragraphe 3.4. Il est important de souligner d'une part que ces tirants d'ancrage sont globalement sur-dimensionnés, par ignorance des chargements qui les sollicitent et que d'autre part il existe des cas de rupture notamment en présence de massifs d'ancrage. On considère le tirant T256 présenté en figure 3a et instrumenté en trois points. Cette instrumentation du quai $\mathrm{T}$ visait à analyser les phénomènes d'adhérence le long du tirant. Les évolutions des efforts centrés sont présentées en figure 3b. On constate une perte importante d'effort par adhérence.

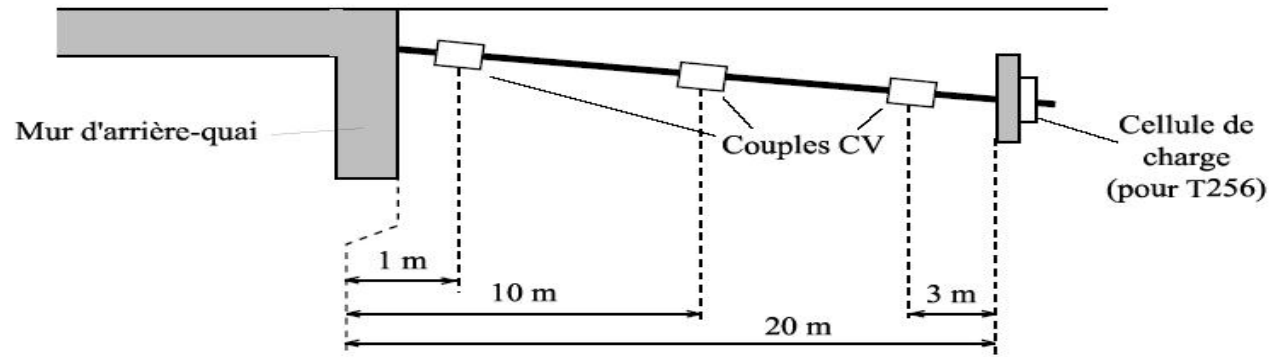

Figure 3a: Caractéristiques d'un tirant instrumenté en trois points (quai T)

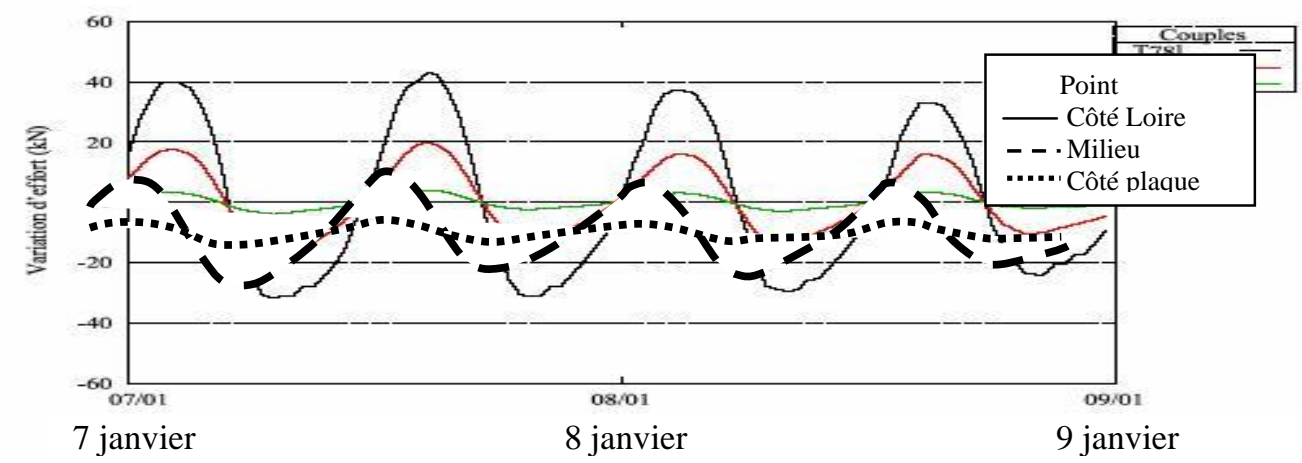

Figure 3b: Variations d'effort [kN] ; tirant instrumenté en trois points (quai T)

La plaque d'ancrage est conçue pour se mettre en butée contre le sol mais rien n'indique qu'elle ne continue pas à se déplacer une fois que la résistance limite du sol est atteinte. Les recherches de solutions technologiques de mesure directe du déplacement n'ont mené à aucun dispositif automatique pour un élément se situant à $4 \mathrm{~m}$ de profondeur. Une possibilité, nécessitant cependant une intervention extérieure pour chaque mesure, est le radar géotechnique. Il s'agit néanmoins d'une technologie émergente, pour laquelle de nombreux développements sont à attendre. Un site test a d'ailleurs été réalisé au Laboratoire Central des Ponts et Chaussées (L.C.P.C.) à Nantes. Au GéM, des tests de faisabilité dans un sable ont été réalisés en partenariat avec la société Calligée, en enterrant des plaques métalliques de $40 \mathrm{~cm} * 40 \mathrm{~cm}$ à une profondeur de $4 \mathrm{~m}$; les plaques qui n'ont pas pu être détectées par le radar ${ }^{1}$.

Cette solution n'a donc pas été retenue et il faut recourir à des modèles de fonctionnement mécanique d'un tirant. La figure $5 \mathrm{~b}$ donne à ce titre des perspectives intéressantes. On présente dans le tableau ci-dessous un comparatif des technologies disponibles pour l'instrumentation d'un tirant ${ }^{2}$. 
Tableau 1: Comparaison des techniques d'instrumentation d'un ouvrage.

\begin{tabular}{|c|c|c|c|}
\hline ie Technolog & Mesure et fonctionnement & Limites & Avantages \\
\hline $\begin{array}{l}\text { Cellule de } \\
\text { charge }\end{array}$ & $\begin{array}{l}\text { Mesure d'effort normal } \\
\text { directe (piézo-électrique ou } \\
\text { hydraulique) à partir de } \\
\text { déformation d'un élément mis } \\
\text { en série dans une structure }\end{array}$ & $\begin{array}{l}\text { Coût élevé. } \\
\text { Positionnem } \\
\text { ent en extrémité - } \\
\text { ici, poau niveau } \\
\text { de la plaque } \\
\text { d'ancrage }\end{array}$ & $\begin{array}{l}\text { Fiabilité } \\
\text { Pérennité }\end{array}$ \\
\hline ètre & $\begin{array}{l}\text { Mesure d'effort normal et } \\
\text { de moment à partir des } \\
\text { déformations du tirant. }\end{array}$ & $\begin{array}{l}\text { Liaison au } \\
\text { tirant à inventer } \\
\text { et valider - } \\
\text { pérennité }\end{array}$ & $\begin{array}{l}\text { Coût faible } \\
\text { Souplesse d'utilisation }\end{array}$ \\
\hline $\begin{array}{r}\text { Jauge } \\
\text { électrique }\end{array}$ & \begin{tabular}{llr}
\multicolumn{1}{c}{ Signal } & \multicolumn{2}{c}{ électrique } \\
proportionnel & à & leur \\
allongement. & & \\
\end{tabular} & \begin{tabular}{l}
\multicolumn{2}{c}{ Surface } \\
plane et propre \\
pour collage. \\
\multicolumn{1}{c}{ Collage "à } \\
chaud" (env. \\
$90 \sim^{\circ} \mathrm{C}$ ) pour \\
éviter tout fluage.
\end{tabular} & $\begin{array}{l}\text { Coût faible, montage en } \\
\text { demi-pont ou } \\
\quad \text { pont complet, ave } \\
\text { compensation } \\
\text { température }\end{array}$ \\
\hline $\begin{array}{l}\text { Capteur à } \\
\text { fibre optique } \\
\text { (CFO) } \\
\text { Capteur à } \\
\text { corde vibrante } \\
(\mathrm{CV}) \text {. }\end{array}$ & $\begin{array}{l}\text { Mesure de fréquence } \\
\text { proportionnelle à l'allongement }\end{array}$ & $\begin{array}{l}\quad \text { Coût élevé } \\
\text { (capteur et } \\
\text { système } \\
\text { d'acquisition) }\end{array}$ & $\begin{array}{l}\text { Fiabilité, taille réduite. } \\
\text { Insensible aux effets } \\
\text { électromagnétiques, } \\
\text { étalonnage non nécessaire. } \\
\text { Coût modéré - fiabilité }\end{array}$ \\
\hline
\end{tabular}

Ces éléments ne doivent pas occulter le fait que l'exigence première vis-à-vis d'une instrumentation est de ne pas affecter la pérennité de l'ouvrage. En particulier, lors qu'il s'agit de capteurs noyés dans le béton ou de capteurs solidaires d'éléments métalliques, la création de points faibles pour les uns et de localisation de corrosion pour les autres doivent être évités. Lorsqu'il s'agit de tirants d'ancrage protégés par galvanisation, le soudage de jauges doit être proscrit. Des cordes vibrantes associées à un procédé par bride ont été choisies (figure 4). Le dispositif de bride, réalisé par l'accouplement vissé de deux demi-brides présente un jeu de montage. Il est donc nécessaire de s'assurer que la rigidité en flexion du système est très importante par rapport à celle des cordes vibrantes. On a montré que la rigidité de l'ensemble bride-tirant de $5000 \mathrm{~N} / \mathrm{m}$ dépassait largement celle la bride seule de 20 $\mathrm{N} / \mathrm{m}$, ce qui valide ce choix.

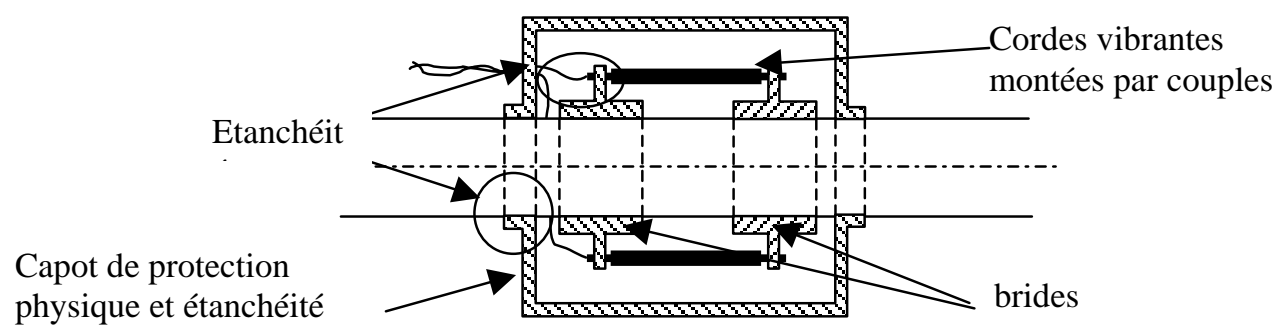

Figure 4: Système de brides des cordes vibrantes sur des tirants. 
En ce qui concerne l'instrumentation, plusieurs objectifs peuvent être visés. Pour cela, le phénomène doit être analysé dans sa localisation et ses variations temporelles ; processus cyclique, monotone... La durée de vie de l'instrumentation doit être étudiée en fonction de ces comportements. Le plus simple est la recherche d'un effet de seuil (dépassement d'un niveau inadmissible). C'est le cas de phénomènes évolutifs de type tassement ou fatigue. Dans ce cas, des capteurs simples et auto-destructifs peuvent être considérés comme suffisants, comme le collage de plaques de verres pour la détection de fissures évolutives : technique utilisée sur les piliers de l'église troglodyte de Saint-Emilion (Gironde-France) en 2001.

La situation la plus complexe est la recherche de la caractérisation d'un processus stochastique qui peut être généré par des effets de surcotes par exemple ou un comportement évolutif long terme, cas de la rotation générale d'un quai ${ }^{3}$. Dans ce cas une acquisition en continu avec fréquence élevée et une pérennité de l'instrumentation doivent être recherchés. En particulier les réductions des points faibles est nécessaire, ceci pour l'ensemble de la chaîne d'acquisition. C'est le cas pour la protection physique de l'instrumen,tation et des câblages. Notamment, une étanchéité parfaite et éventuellement une signalisation d'enfouissement doivent être adoptées. Un autre exemple est le choix du nombre de fils dans les câblages de ponts complets de jauges : l'utilisation d'un montage six fils permet un recâblage ou une évaluation de la distance le séparant de la centrale d'acquisition en cas de perte de repérage.

\subsection{Recherche de redondance}

Lors d'une instrumentation de structures, la redondance d'informations doit être recherchée d'autant plus que les incertitudes sur le comportement et/ou le chargement de l'ouvrage sont méconnus. Elle s'accompagne d'un surcoût si ce seul poste est étudié. Dans le cas d'une approche globale d'IMIA, l'incertitude sur certaines informations isolées et les exigences de sécurité de l'ouvrage peuvent conduire à une maintenance pro-active coûteuse et non-optimisée ${ }^{4}$.

Au niveau d'un comportement long terme, la mesure de déplacements en plus des efforts peut constituer un élément important de redondance. Le choix de plusieurs capteurs permet de valider des choix technologiques (voir figure 3a).

Au niveau du comportement système de l'ouvrage, une redondance est à envisager si les comportements composants sont accompagnés d'aléas (voir figure 2). A titre d'exemple, une instrumentation globale d'un quai $(\mathrm{T})$ a été réalisée en 12 tirants - 10 tirants instrumentés en partie courante, soit 1 sur 4, compromis entre le coût global et la quantité d'informations et 2 tirants aux extrémités amont et aval en trois points pour l'étude des effets de bord et du frottement. En revanche sur le quai D, l'absence de frottement sol tirant a permis une redondance moindre. La redondance d'information et la complémentarité peuvent être recherchées aussi au niveau d'un parc d'ouvrages.

\subsection{Sécurisation des données et interface gestionnaire}

En instrumentation, l'archivage et la sécurisation des données sont essentielles. De même des interfaces gestionnaires de type tableau de bord en temps réel permettent le suivi des structures et alertent sur des défaillances. L'ensemble de ce dispositif est aujourd'hui accessible grâce aux services de OXAND.net. 


\section{Conclusions}

L'instrumentation des ouvrages est actuellement un enjeu majeur afin de cerner leur comportement en service et de prédire d'éventuelles défaillances dans le but final d'optimiser les inspections, maintenance et réparation. Cet article se concentre sur une partie d'un parc d'ouvrages et indique les différents enjeux technologiques et stratégiques qui doivent être abordés.

\section{Références}

1 Verdure L., "Test de faisabilité d'une méthode de mesure de déplacement de plaques d'ancrage géotechnique par radar géotechnique", Institut de Recherche en Génie Civil et Mécanique, Rapport interne $\mathrm{n}^{\circ} 011$ ins-01 - 2001, 6 pages.

2 Verdure L., Casari P., Schoefs F., "Suivi en service d'un quai sur pieux par mesure mécaniques ", Méthodes d'évaluations non destructives pour le génie civil, Journées scientifiques ENDGC2, Bordeaux, 8-9 novembre 2001.

3 Verdure L., Casari P., Wielgosz C., " Joint use of instrumentation and probabilistic modeling applied to acontainer wharf", Bridges and special structures, Proceeding of 9th International Conference on Applications of Statistics and Probability in Civil Engineering, (I.C.A.S.P’03), july 6-9 2003, San Francisco, U.S.A, vol.2, ISBN 905966005 4, pp. 17331740, Millpress Rotterdam 2003.

4 Schoefs F., Bernard O., "Probabilistic updating of corroded reinforced concrete pipes for maintenance planning optimisation", Proceeding of 3rd International Workshop on Life-Cycle Cost Analysis and Design of Civil Infrastructure Systems (L.C.C’03), Lausanne, 24-26 march 2003, 12 pages. 